\title{
Kullback-Leibler Dissimilarity of Markov Models for Phylogenetic Tree Reconstruction
}

\author{
Tuan D. Pham ${ }^{1,2}$, Denis I. Crane ${ }^{3,4}$, David 'Tannock ${ }^{1}$, and Dominik Beck ${ }^{1}$ \\ ${ }^{1}$ School of Computing and Information Teclnology \\ ${ }^{2}$ Institute for Integrated and Intelligent Systems \\ ${ }^{3}$ Sclool of Biomolecular and Biomedical Science \\ ${ }^{4}$ Eskitis Institute for Cell and Molecular Therapies \\ Griffith University, Nathan Campus, QLD 4111, Australia \\ E-mail: t.phanggriffith.edu .au
}

\begin{abstract}
In this paper, we introluce the Kullback-Leibler dissimilarity measure of Markov-chain models for unaligned DNA sequences with application to the phylogenetic troc reconstruction of complete mammalian mitochondrial genomes. The tree obtained by our approach is generally in agreement with those obtaincd from other methods. Our proposed method is computationally efficient and very casy for computer implementation.
\end{abstract}

\section{INTRODUCTION}

The study of similarity/dissimilarity comparison of DNA gonomic sequences is a challenging and inportant area of conputational biology and bioinformaties [8]. Two distinct bioinformatic methodologies for studying the sinularity/dissimilarity of scquences arc known as alignment-based and alignment-free methods. The scarch for optimal solutions using multiple scruence alignment methorls [15] encounters difficulty in computational aspect whon applied to large biological databases. Alignment-fice sequence analysis represents a potential means to these critical lintitations of sequence analysis by aligmment.

One of the most recont reviews [16] on published methods for alignment-frec sequence comparison of biological sequences reports several concepts of distance measures such as the Euclidean distance [2], Euclidean and Mahalanobis distances [17], Markov chain models and Kullback-Leibler discropancy [17], cosine distance [13], Kolmogorov complexity [7], and chaos theory [1]. Our present work bears some strong similarity to that by $W_{11}$ ct al. $[17]$ in which statistical measures of DNA sequence dissimilarity as the Mahalanobis distance and standardized Euclidean distance under Markov chain model of base composition, as well as the extended Kullback-Leibler discrepancy (KLD). The KLD extended by Wu et al. [17] was computed in terms of two vectors of relative frequencies of $n$-workls over a sliding window from two given DNA sequences. In contrast, we have derived [10] a probabilistic distance betwcen two seruences using a symmetrized version of the KLD, which directly compares two Markov models built for the two corresponding biological sequences, for database search of sequence relatedness. We now apply this KLD of Markov models for the pliylogenctic tree reconstruction of whole mammalian mitochondrial genomes.

\section{KLD OF MARKOV MODELS FOR UNALIGNED DNA SEQUENCES}

Let $A=\left[a_{i j}\right]$ denote the state transition probability matrix of a discrete Markov process. Each state transition probability $a_{i j}$ is defined as

$$
a_{i j}=P\left[q_{n}=S_{j} \mid q_{n-1}=S_{i}\right], 1 \leq i, j \leq N
$$

where $q_{t_{n}}$ stands for the actual state at time $t_{n}$ $(n=1,2, \ldots), S_{j}$ a state $j$ of a set of $N$ distinct states. In the context of DNA sequences, the number of states $N=4$ which correspond to the four uucleotide symbols $\{a, c, g, t\}$.

The state transition probabilities aro subject to

$$
\begin{gathered}
a_{i j} \geq 0 \forall i, j \\
\sum_{j=1}^{N} a_{i j}=1 \forall i
\end{gathered}
$$

Also let $\pi=\left\{\pi_{i}\right\}$ be the initial state transition distribution where

$$
\pi_{i}=P\left(q_{t_{1}}=S_{i}\right), 1 \leq i \leq N
$$

This Narkov chain involves two probabilistic measures $A$ and $\pi$, that can be denoted in a compact form as

$$
\lambda=(A, \pi)
$$

What we have presented above is the first-order Markov model. The higher-order Markov models can be determincel as follows. Let $\left\{\pi_{j}^{(n=1)}\right\}(j=$ $1,2, \ldots, N)$ be the absolute (initial) probabilities that the system is in state $S_{j}$ at $t_{1}$. 


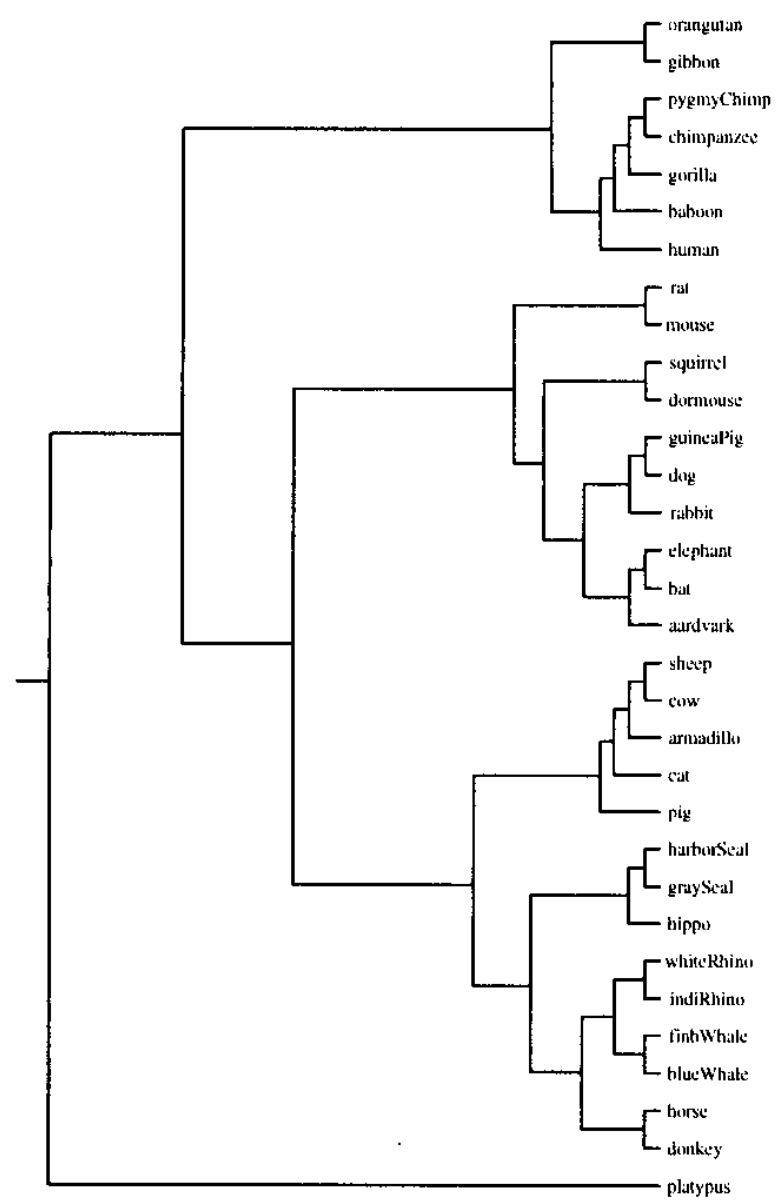

Fignre 1. Plylogenetic tree reconstruction using KLD of MM

Given the parameters $\left\{\pi_{j}^{(1)}\right\}$ and $A$ of a Markov chain, the absolute probabilities after a specified number of transitions ( $n$-order) are determined as follows.

$$
\pi_{j}^{(n)}=\sum_{i} \pi_{i}^{(1)} a_{i j}^{(n)}
$$

where $a_{i j}^{(n)}$ is the $n$-step or n-order transition probability given by the recursive formula

$$
a_{i j}^{(n)}=\sum_{k} a_{i k}^{(n-m)} a_{k j}^{(m)}, 0<m<n
$$

Let $\lambda_{1}=\left(A_{1}, \pi_{1}\right)$, and $\lambda_{2}=\left(A_{2}, \pi_{2}\right)$ be two Markov models of the two bio-sequences, where each model is constructed by the observed symbols of each corresponding DNA sequence. Our interest is to find a similarity or dissinilarity measure between two Markov models $\lambda_{1}$ and $\lambda_{2}$. A woll-known dissimi- lanity measure between two probability distributions is the Kullback-Leibler divergence (KLD) [4].

Let $P_{1}$ and $P_{2}$ be two probability distributions on a universe $X$, the Kullback-Leibler divergence or the relative entropy, denoted as $H\left(P_{1}, P_{2}\right)$, of $P_{1}$ with respect to $P_{2}$ is defined by the Lebesguc integral

$$
H\left(P_{1}, P_{2}\right)=\int_{X} \frac{d P_{1}}{d P_{2}} \log \frac{d P_{1}}{d P_{2}} d P_{2}
$$

The discrete version of the KLD defined in (8) is

$$
H\left(p_{1}, p_{2}\right)=\sum_{x \in X} p_{1}(x) \log \frac{p_{1}(x)}{p_{2}(x)}
$$

Given two Mar'kov models, we now can define a probabilistic distance between two secquences, denoted by $d\left(\lambda_{1}, \lambda_{2}\right)$, as

$$
d\left(\lambda_{\mathbf{l}}, \lambda_{2}\right)=1-\exp \left[D_{s}\left(\lambda_{1}, \lambda_{2}\right)\right]
$$




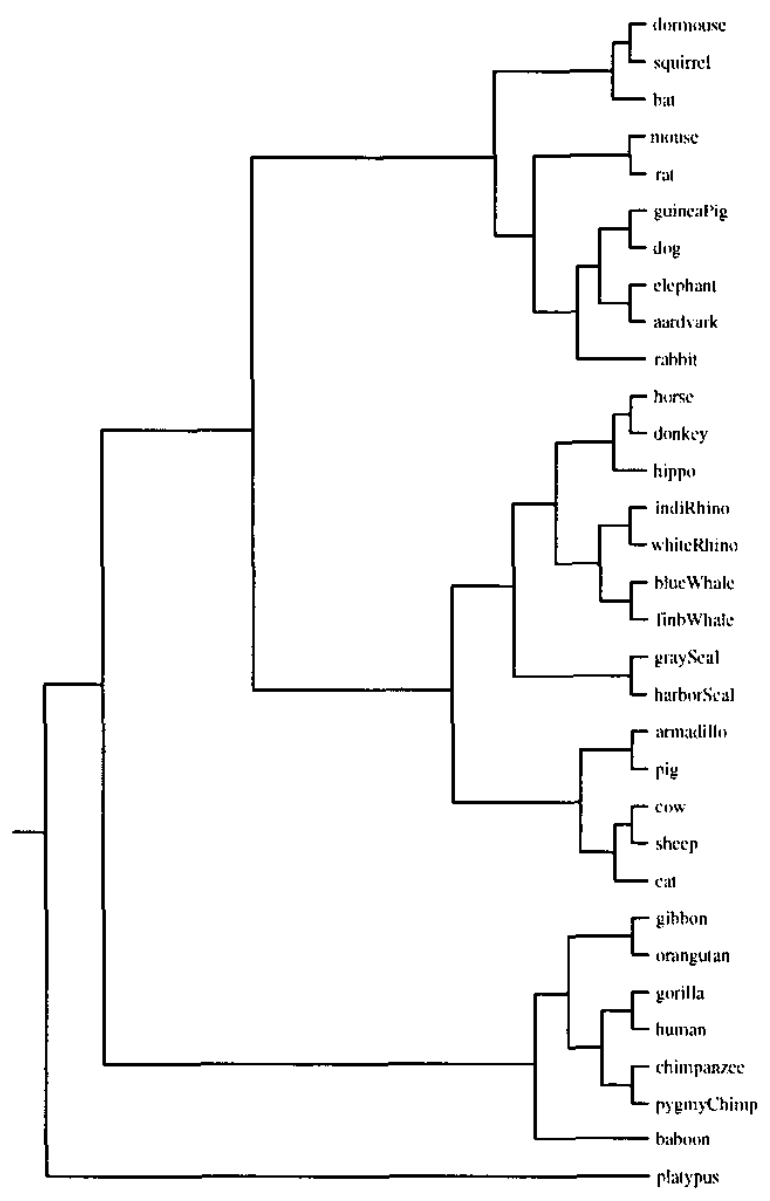

Figure 2. Plylogenetic tree reconstruction using 3-word conposition approach

where $D_{s}$ is the symmetrized version of the approximate KLD of $\lambda_{1}$ and $\lambda_{2}$, which is expressed as

$$
D_{s}\left(\lambda_{1}, \lambda_{2}\right)=\frac{D\left(\lambda_{1}, \lambda_{2}\right)+D\left(\lambda_{2}, \lambda_{1}\right)}{2}
$$

in which $D\left(\lambda_{1}, \lambda_{2}\right)$ is the empirical KLD between $\lambda_{1}$ and $\lambda_{2}$, which was originally introduced by Juang and Rabiner [6] using the Nonte Carlo sinulations. The models are assumed to be ergodic, laving arbitrary observation probability distributions; and the dissimilarity is defined as the mean divergence of the observation sample. This approximate KLD is given by $[11]$

$$
D\left(\lambda_{1}, \lambda_{2}\right)=\frac{1}{T_{2}^{2}} \log \frac{P\left(O_{\lambda_{2}} \mid \lambda_{1}\right)}{P\left(O_{\lambda_{2}} \mid \lambda_{2}\right)}
$$

where $O_{\lambda_{2}}=\left(o_{1} O_{2} \ldots o_{T_{2}}\right)$ is a sequence of observations generated by model $\lambda_{2}$, and $T_{2}$ is the lengtl of sequence $O_{\lambda_{2}}$.
Finally, the probability of the observation sequence $O=\left\{o_{t}, t=1, \ldots, T\right\}$, given a Markov model $\lambda$ can be ovaluated as

$$
\begin{aligned}
P(O \mid \lambda) & =P\left(o_{1}, \ldots, o_{T} \mid \lambda\right) \\
& =P\left(o_{1}\right) \ldots P\left(o_{t} \mid o_{t+1}\right) \ldots P\left(o_{T-1} \mid o_{T}\right) \\
& =\pi_{\left(q_{1}=o_{1}\right)} \ldots a_{q_{t,}, q_{t_{2+1}}} \ldots a_{q_{t_{T-1}} q_{r_{T}}}
\end{aligned}
$$

\section{RESULT}

The proposed method was used to reconstruct the phylogenctic tree of 32 complete mammalian mitochondrial DNA genones. The evolutionary relationship betwcen species as based on sequence analysis of mitochondrial genomes was studied by Reyes et al. [12], then $\mathrm{Li}$ et al. [7], and Otu and Sayood [9]. 
However, tliese analyses have led to diverse ontcomes and the relative designations remain controversial.

The mtDNA sequences were obtained from the public-domain database of the National Center for Biotechology Information (NCBI) (www.ncbinhmili.gov/Entrez/). The genomes consist of members of the primates, rodents, and ferungulates. The primates include human (NC_001807), climpanzee (NC_001643), pygmy chimpanzee (NC_001644), baboon (NC_001992), gorilla (NC_001645), orangutan (NC_001646), and gibbon (NC_002082). The rodent group includes rat (NC_002083), mouse (NC_005089), dormouse (NC_001892), squirrel (NC_002369), guinea pig (NC_000884), and fruitbat (NC_002009). The ferungulates include cat (NC_001700), dog (NC_002008), harbor seal (NC_001325), gray seal (NC_001602) white rhino (NC_001808), indian rhino (NC_001779), blue whale (NC_001601), finback whale (NC_001321), pig (NC_000845), armadillo (NC_001821), aardvard (NC_002078), elepliant (NC_000934), cow (NC_001567), sheep (NC_001941), donkey (NC_001788), lıorse (NC_001640), hippopotamus (NC_000889), and rabbit (NC_001913). The outgroup consists of platypus(NC_000891).

We used our proposed metlod to calculate the distance matrix for the above 32 complete mtDNA genomes. For the Markov model, the trausition probabilities are of third order where the solution converges. The result plotted by the NJ program in the PHYLIP package [5] is shown in Figure 1. The overall structure of the phylogenetic tree obtained by our proposed approach agrees with those obtained by Reyes et al. [12], Li et al. [7], and Otu and Sayood $[9]$. However, the result of the proposed method gromps the rodents with the ferungulates; whereas the result obtainerl by Otu and Sayood groups the prinkates with the ferungulates.

We also used the word composition approach [16] based on three-word composition statistics to analyze the same data set and the result obtained, which is shown in Figure 2, is similar to that by oul proposed method: both methods group the rodents with the fer'ungulates.

In particular, with the analysis of the rodent group, our method, like the other methods, clusters dormouse with squirrel (nonmurid rodents), and mouse with rat (murid rodents). On method and the word composition approach do not place tle guinea pig, whose position still remains an open question [3], with the two well-supported rodent clades - nonmutid rodents and murid rodents. However, the study of mammalian phylogeny is still controversial largely clue to conflicting findings regarding tlie pliylogeny of eutherian order's $[3,9]$.

\section{CONCLUSION}

We have presented a KLD measure of Markov models for genome phylogenetic tree reconstruction. The experiment on a data set of 32 whole mtDNA genomes has shown the effectiveness of the proposed method. Our proposed metlod is simple for computer implementation as a new tool for comparative genonics.

\section{References}

[1] Almeida,J.S., Gatrico.J.A., Maretzek,A., Noble,P.A., and Fletcher, M. (2001) Analysis of genomic sequetrees by chaos gane representation. Bioinformatics, 17, 429-437.

[2] Blaisdefl,B.E. (1986) A measure of the similarity of sets of sequences not requiring sequence álignment. Proc. Natl. Acad. Sci. USA, 83, 5155-5150

[3] Cao, Y., A. Janke, P.J. Waddell, M. Westerman, O. Takenaka, S. Murata, N. Okada, S. Paabo, and M. Jasegawa, Conflict anong individual mitochondrial proteins in resolving the pliylogeny of eutherian orders, $J$ Mol. Evol., 47 (1998) 307-322.

[4] Cover, T.M. atud Thomas, J.A. (1091) Elements of Information Theory. John Wiley and Sons, New York, 1991

[5] Felsenstein, J. (1903) PHYLIP (Phylogeny Inference Package), version $3.5 c$, distributed by the author, Department of Genetics, University of Washington, Seattle.

[6] Juang, B.H., and Rabiner, L.R., a probabilist ic distance measure for hidden Markov models, $A T$ T Tech. J., 6.1 (1985) 391-408.

[7] Li,M., Badger,J.H, Chen,X., Kwong,S., Kearney,P. and Zhang, II. (2001) An information-based sequence distance and its application to whole mitochondrial genome phylogeny, Bioinformatics, 17, 1.19-15.1.

[8] Miller,W. (2001) Comparison of genomic DNA sequences: solved and insolved problems. Bioinformatics, 17, 391-397.

[9] Otu, H.H., and K. Sayood, K., A new sequence distance measure for phylogenetic tree construction, Biainformatics, 19 (2003), $2122-2130$.

[10] Pham, T.D., and Zuegg, J., A probabilistic measure fu aligmment free sequence comparison, Bioinformatics, iccepted.

[11] Rabiner, L.R. (1989) A tutorial on hidden Markov models and selected applications in speecli recognition. Proc. IEEE, $77,257-286$

[12] Reyes, A., Gissi, C., Pesole, G., Catzeflis, F.M1., and Saccone, C., Where do roclests fit? Evidence from complete mitochondrial genome of Sciurus vulgaris, Mol. Biol. Evol, 17 (2000) $979-983$

[13] Stuart,G.W., Moffet t,K. and Baker,\$. (2002) Int egrated gene and species pliylogenies from unaligned whole genone protein sequences. Bioinformatics, 18, 100-108.

[14] Taha, H.A. (1982) Operations Research: An Introduction. Macmillan, New York.

[15] Thompson,J.D., Iliggins, D.G. and Gibson, T.J. (1994) CLUSTAL W: improving the sensitivity of progressive multiple sequence alignment through sequence weighting, position-specific gap penalties and weiglt matrix choice. Nucleic Acids Res., 22, 4673-4680.

[16] Viıga,S., and Almeida,J. (2003) Aligmment-free seruelıce comparison - $A$ review, Bioinformatics, 10, 513-523.

[17] Wu,T.J., Hsieh,Y.C. and Li,L.A. (2001) Statistical Ineasures of DNA dissimilarity under Markov chain models of base composition. Biometrics, 57, 441-4:18. 